\title{
Der ältere Handpatient
}

\author{
Rebecca Groth
}

Die Handanatomie verändert sich mit den Lebensjahren eines Menschen kaum. Mit dem Alterungsprozess durchläuft der Körper aber physiologische Prozesse, durch die sich die Beschaffenheit von Muskeln, Bindegewebe und Knorpel anpasst. Diese Strukturveränderungen müssen neben der Alterung des körpereigenen Stoffwechsels und der neuronalen Ansteuerung in der Behandlung der geriatrischen Hand Berücksichtigung finden.

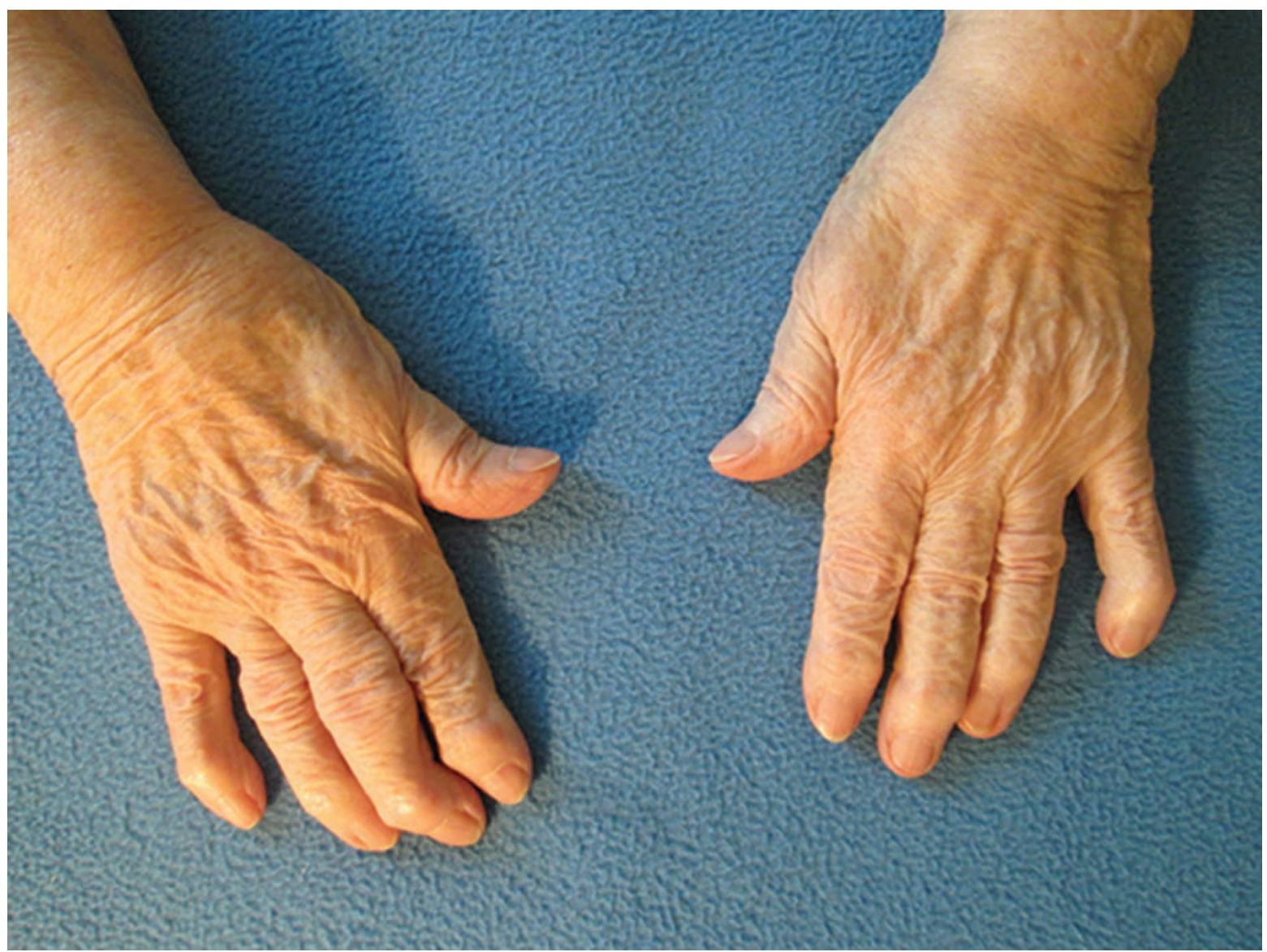

Physiologisch gealterte Hände einer 97-jährigen Dame. (Quelle: R. Groth)

\section{Physiologische Alterungsprozesse}

Mit zunehmenden Jahren stellt sich die Anpassungsfähigkeit der Körpersysteme auf plötzliche Ereignisse um. Dadurch sind ältere Menschen schlechter in der Lage, adäquat auf physischen und psychischen Stress, wechselnde Umgebungen oder Anforderungen zu reagieren. Dies beruht auf Umgestaltungen im muskuloskelettalen, neuromuskulären, sensorischen, kognitiven sowie im Herz-Kreislauf-System.

\section{Veränderungen im muskuloskelettalen System}

Sie betreffen alle Gewebearten des Körpers und werden nach ihren Auswirkungen unterteilt: Sarkopenie (= Verlust an Muskelmasse), Osteoporose (= verminderte Knochendichte), Pergamenthaut (= sehr dünne, wenig belastbare Haut) sowie der Elastizitätsverlust in Knorpel und Bindegewebsstrukturen. Zurückzuführen sind diese Erscheinungen auf einen Verlust der Zellen und eine 
abnehmende Syntheseaktivität dieser bei reduzierter Qualität der produzierten Matrixbestandteile [2]. Somit unterliegen die Gewebe einer veränderten Ernährungslage und Belastbarkeit.

Der Verlust an Muskelmasse und Kraft geht mit Aktivitätseinbußen und Einschränkungen der Handfunktion einher und ist daher aus handtherapeutischer Sicht besonders wichtig. An der Hand sind insbesondere die Mm. interossei und der Thenar betroffen, was zu einer Verminderung der Griffkraft führt [4]. Dies scheint ein Prozess zu sein, der sich auch auf die kortikale Dedifferenzierung zurückführen lässt. Gerade die Ansteuerung der intrinsischen Handmuskulatur bedarf einer genaueren kortikalen Kontrolle und gezielten Ansteuerung als die weit gröbere Ansteuerung der extrinsischen Muskulatur.

Die Minderung der Handkraft ist zudem durch die verringerte Hautspannung bei älteren Menschen bedingt. Der Wasserverlust der Haut lässt die Hautoberfläche weniger Kontakt an Objekten finden, weswegen diese den Menschen leichter aus der Hand rutschen [1]. Der Wasserverlust der Subkutis führt außerdem zu einer verminderten Elastizität und Spannkraft der Haut. Das damit einhergehende erhöhte Verletzungsrisiko der Haut wird durch Nebenerkrankungen wie Diabetes mellitus aufgrund des verschlechterten Stoffwechsels noch gesteigert. Ein weiterer Risikofaktor für die Ausprägung einer Pergamenthaut besteht in Dauermedikationen mit Cortison oder Marcumar. Dies hat im Weiteren auch Auswirkungen auf die Wundheilung. Sie läuft bei der Altershaut grundsätzlich zwar ab wie bei jüngerer Haut, aber auf erneuten Stress (z. B. beim Verbandwechsel) reagiert sie mit neuerlichen Verletzungen.

Durch die Verminderung der Kraft wird die Minderung der Knochendichte begünstigt, die durch den veränderten Hormonstoffwechsel hervorgerufen wird. Die Osteoporose ist also sowohl Folge der veränderten Stoffwechsellage als auch einer Minderung der auf den Knochen einwirkenden Kräfte [2].

Auch der Knorpel ist auf regelmäßige Belastung angewiesen, da permanente Druckveränderungen seine Ernährung gewährleisten. Bei Belastungsmangel wird weniger Grundsubstanz synthetisiert, der Knorpel kann weit weniger Wasser binden und sein kollagenes Netzwerk wird dadurch bedingt verformbarer. Je verformbarer der Knorpel wird, desto größer ist die Belastung für die einzelnen kollagenen Fibrillen, wodurch Schädigungen des Knorpels wie beispielsweise Arthrosen entstehen [2].

\section{Veränderungen im neuromuskulären System}

Mit zunehmenden Lebensjahren kommt es zu einem stetigen Verlust an Kraft, Bewegungsgeschwindigkeit und Koordination. Nervenleitgeschwindigkeit und die Sensoraktivität verringern sich bei einer gleichzeitigen Erhöhung der Erregungsschwelle. Hinzu kommen altersbedingte Verluste in der kortikalen Kontrolle und Ansteuerung [1]. Diese grundlegenden Prozesse bedingen eine verlangsamte und ungenauere Ansteuerung der Muskulatur, was sich zum einen in einer Kraftminderung, aber auch einer Minderung der feinen Ziel- und Greifmotorik niederschlägt. Ein weiterer wichtiger Aspekt betrifft in diesem Sinne die Ansteuerungsgeschwindigkeit, wie sie bei Sturzund Stützreflexen benötigt wird, um sich noch rechtzeitig abfangen zu können.

Durch die physiologischen Abbauprozesse hat ein 80Jähriger bereits fast die Hälfte seiner Motoneuronen, motorischen Einheiten, Muskelfasern und Muskelmasse verloren [4]. Dies führt zu Veränderungen in der Aktivierung der Muskulatur. So weisen ältere Erwachsene eine stärkere Kokontraktion der Antagonisten in ihren Bewegungsabläufen auf. Daraus resultiert zwar eine bessere Stabilisation der Gelenke, gleichzeitig aber auch eine schlechtere Kraftübertragung.

\section{Veränderungen im sensorischen System}

Die motorischen Fähigkeiten älterer Menschen werden auch durch verminderte sensorische Rückmeldungen, wie Defizite im taktil-sensorischen oder auch dem propriozeptiven System, negativ beeinflusst. Dies betrifft zum einen den taktilen Schwellenwert, ab dem eine Berührung wahrgenommen werden kann, was zu einer verminderten räumlichen Genauigkeit der motorischen Leistungen führt. Zum anderen bedingt eine Abschwächung der propriozeptiven Rückmeldungen eine Abschwächung der Hand-Hand-Koordination und auch Ungenauigkeiten im Krafteinsatz. Dabei ergeben sich durch Veränderungen der Durchblutung, Abnahme der Rezeptorendichte, altersbedingte Erkrankungen oder Traumata individuelle Unterschiede in der Rezeptorendichte in der Haut und im Gewebe [4]. Hinzu kommt die perzeptive Nutzung der betroffenen Strukturen. Zu den taktilen Defiziten reihen sich Beeinträchtigungen visueller Prozesse. So führt eine verminderte Sehleistung zu Einschränkungen der feinen Greif- und Zielbewegungen. Sie sind eine Schlüsselkomponente für die Verringerung motorischer Koordination und Funktion. Dazu gehören eine verminderte Sehschärfe, Anpassung, Farbdifferenzierung, Lichtempfindlichkeit, Tiefenwahrnehmung, beeinträchtigte Hand-Auge-Koordination sowie die Anpassung an Hell und Dunkel, die die motorischen Leistungen der Hand beeinflussen können.

In Bezug auf Verletzungen und Veränderungen der Physiologie stellt auch die Schmerzwahrnehmung älterer Patienten eine Herausforderung dar. So stellte Gibson in einer Metaanalyse eine Erhöhung der Schmerzschwelle mit zunehmendem Alter fest [5]. Die somatosensorischen Schwellenwerte für nicht schädliche Reize sind im Alter erhöht, wohingegen die Druckschmerzschwelle absinkt und die Schmerzschwelle für Hitzereize 
unverändert bleibt [9]. Zu diesen altersphysiologischen Veränderungen kommt der veränderte Umgang mit Schmerzen. Ältere Patienten geben Schmerzen oftmals erst sehr spät an, weil sie der Meinung sind, diese seien Teil des normalen Alterungsprozesses [8].

\section{Veränderungen im kognitiven System}

Die kognitive Ansteuerung differenzierter Bewegungen weist im Vergleich zu jungen Probanden Unterschiede auf. So lässt sich mit zunehmendem Lebensalter eine Abschwächung der Lateralität des Gehirns beobachten. Wo jüngere Probanden (20-32 Jahre) bei einer feinmotorischen Aufgabe eindeutig ihre dominante Hemisphäre aktivieren, lässt sich bei Älteren (50-77 Jahre) eine seitengleiche kortikale Reaktion beobachten. Dies kann auf kompensatorische Mechanismen des Gehirns aufgrund kortikalen Abbaus sowie auf eine verminderte Fähigkeit zur Differenzierung zurückgeführt werden. Der ältere Erwachsene ist in seinen Fähigkeiten, spezialisierte neuronale Mechanismen zu aktivieren, eingeschränkt [10].

\section{Veränderungen im Herz-Kreislauf-System/ Stoffwechsel}

Mit zunehmendem Alter verändern sich sowohl die Zusammensetzung des Körpers als auch die Leistungsfähigkeit der inneren Organe. So arbeiten beispielsweise Leber und Niere mit den Jahren langsamer. Das HerzKreislauf-System vermindert zwar nicht seine Leistungsfähigkeit, passt aber Frequenz und Blutdruck langsamer auf veränderte Belastungen an. Dazu kommt der verminderte Wassergehalt des Körpers, der bei 30-40\% statt bei ca. $50 \%$ liegt. Im Gegensatz dazu erhöht sich im Alter der Körperfettanteil um etwa ein Drittel [3].

Medikamente können nicht mehr einfach nach dem Gesamtkörpergewicht berechnet werden, sonst würden sich im Gewebswasser wirksame Substanzen in ihrer Konzentration verstärken und die sich im Fett einlagernden Substanzen länger auswirken. Die verminderte Leistung der ausscheidenden Organe verändert die Wirkstoffkonzentration im Körper und deren Abbauzeiten. Um diesen Wechselwirkungen des alternden Menschen gerecht werden zu können, entwickelten Geriater die sogenannte Priscus-Liste [7], auf der problematische Medikamente für den alternden Körper ebenso wie besser verträgliche Substanzen aufgeführt sind.

\section{Altersphysiologische Veränderungen sind beeinflussbar}

Die Ausprägung dieser altersphysiologischen Veränderungen hängt nicht nur von Erbanlagen und Kontextfaktoren ab, sondern ganz wesentlich von der Aktivität des Menschen. So lässt sich an den häufig eingesetzten Muskelgruppen ein geringerer Abbau und Kraftverlust feststellen. Ebenso bleiben sensorische Fähigkeiten bei durchgehender intensiver Nutzung in dem spezifischen Nutzungsbereich erhalten, während in Bereichen geringerer Nutzung die Rezeptorendichte zurückgeht [14].

Die über die gesamte Lebensspanne hinweg laufenden Anpassungen des Organismus neigen sich in einem stetigen Wechsel zwischen Auf- und Abbauprozessen zwar immer mehr den Abbauprozessen entgegen. Das heißt allerdings nicht, dass der alternde Körper keine Aufbauprozesse mehr bewältigen kann. Es bedarf dazu jedoch weit stärkerer und andauernderer Bildungsreize als in jüngeren Jahren. So wurde in einer Studie festgestellt, dass blinde ältere Menschen eine bessere Empfindlichkeit in dem Finger aufweisen, den sie zum Lesen der Blindenschrift benötigen. Allerdings nicht an den anderen Fingern [14].

Somit sind aber auch Alterungsprozesse wie Sarkopenie und Osteoporose Prozesse, die durch ausreichende Bildungsreize beeinflussbar sind. So besteht sowohl für den Knochen als auch für die Muskulatur und den Knorpel der Bedarf an regelmäßiger Belastung, um den Abbauprozessen entgegenzuwirken. Bei verminderter Belastung des muskuloskelettalen Systems, wie sie im Alter häufiger zu beobachten ist, werden die altersphysiologischen Abbauprozesse verstärkt, da die benötigten physiologischen Bildungsreize ausbleiben. Es bedarf also einer gezielten und regelmäßigen Belastung des Gesamtsystems. Je deutlicher und gezielter die Trainingsreize auf die Strukturen sind, desto wahrscheinlicher schützen sie vor Folgeverletzungen durch Stürze und andere Unfälle im Alltag älterer Menschen. Einmalig ein Programm zur Sturzprävention zu durchlaufen nutzt aus diesen Gründen wenig, da es neben dem gezielten Üben auch auf die Regelmäßigkeit ankommt. Gerade ein solch regelmäßiges Üben lässt sich in den Alltag nur durch eine gute Patientenedukation unter Einbezug der Angehörigen erreichen.

\section{Grundsätze für die handtherapeutische Herangehensweise}

Aus diesen Grundlagen ergeben sich die wesentlichen Änderungen in den handtherapeutischen Techniken, verglichen mit der Handtherapie bei jüngeren Menschen: So bedingt der verminderte und verlangsamte Stoffwechsel eine längere Vorbereitungszeit für die zu behandelnden Strukturen, um die Versorgungslage vor der Behandlung zu optimieren.

\section{Vorbereitung des Gelenks}

Als adäquate Vorbereitung für das Bindegewebe der Kapsel sollte ein ausführliches Bewegungsprogramm ohne Widerstand durchgeführt werden. Dies kann im warmen Raps ( $\triangleright$ Abb. $\mathbf{1}$ ) ebenso geschehen wie manualtherapeutisch mit einer sanften Push-and-Pull-Technik. 


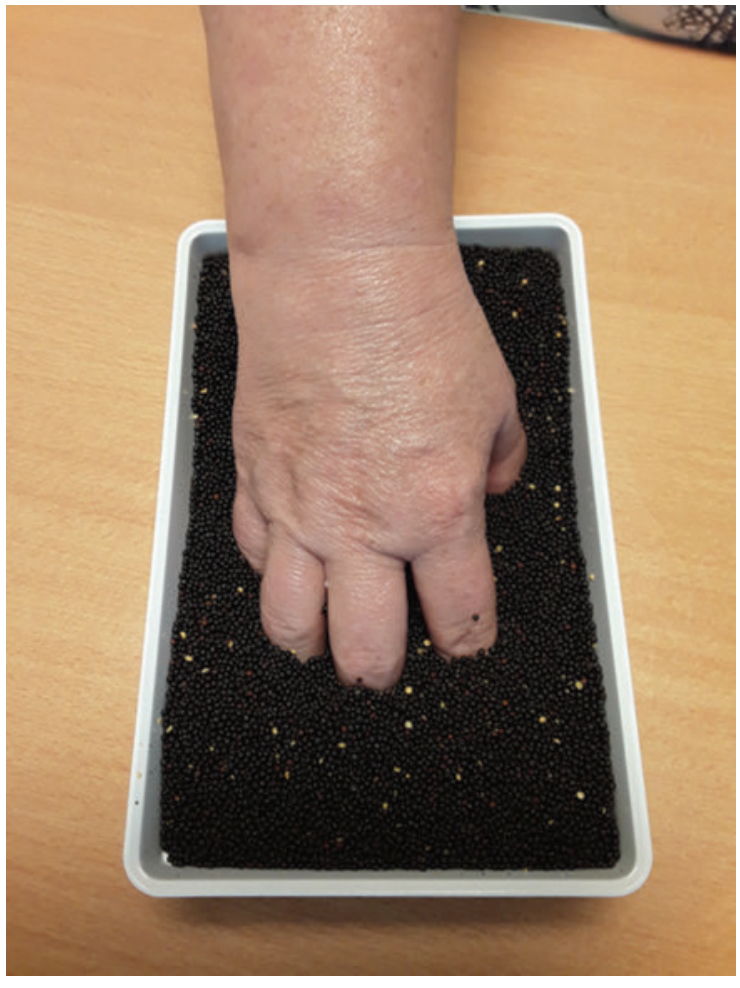

-Abb. 1 Schmerzfreie Bewegungsübungen im warmen Rapsbad. (Quelle: S. Wittek)

Die Dauer wird so gewählt, dass eine leichte Mehrdurchblutung auf der Haut sichtbar ist oder der Patient ein weicheres Bewegungsgefühl angibt. Ist der Patient zur Differenzierung nicht in der Lage, kann eine ausreichende Vorbereitung der Kapsel an einer Verbesserung des Gelenkspiels erkannt werden. In jedem Falle lässt sich die Vorbereitungszeit nicht in einer genauen Zeit festlegen. Sie bemisst sich individuell nach den Anforderungen des Bindegewebes des Patienten. Über die sanften Bewegungsübungen ohne Widerstand wird nebenbei auch eine Erwärmung der Muskulatur erreicht sowie eine perzeptive Aufmerksamkeitsfokussierung auf den zu behandelnden Bereich.

Für eine optimierte Knorpelversorgung bedarf es regelmäßiger Druckwechsel im Gelenk. Diese können manualtherapeutisch erreicht werden, aber auch über Bewegungen oder isometrische Anspannungen gegen leichten bis mittleren Widerstand. Der Widerstand für diesen Part der Vorbereitung bemisst sich an der individuellen Leistungsfähigkeit aller beteiligten Strukturen. Er darf in keinem Falle Schmerzen verursachen. Der Patient darf eine leichte Anstrengung verspüren.

\section{Umgang mit der Altershaut}

Hinzu kommt die Notwendigkeit zum vorsichtigen und achtsamen Umgang mit der Altershaut, um Verletzungen zu vermeiden ( $\mathbf{A}$ bb. 2). Pflegen und Cremen sind wichtig. Außerdem sollten keine Zugbelastungen oder

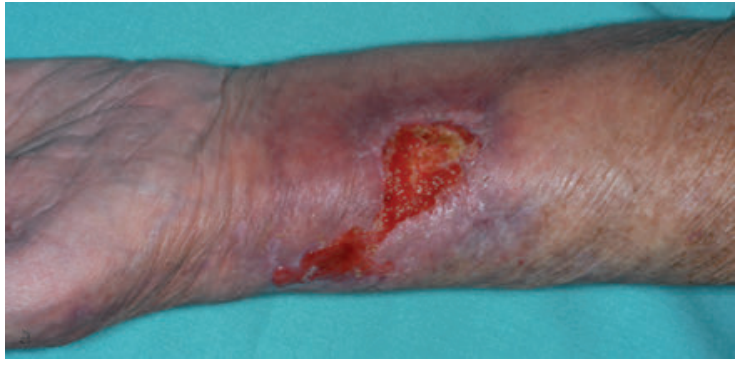

Abb. 2 Verletzung der Pergamenthaut. (Quelle: Tautenhahn J, Piatek S. Polyurethanschaumstoffe / Hydropolymere. In: Lippert H, Hrsg. Wundatlas. 3. vollständig überarbeitete und erweiterte Auflage. Stuttgart: Thieme; 2012. doi:10.1055/b-002-35728)

Scherbewegungen bei therapeutischen Techniken auf die Haut einwirken. Dazu gehört auch, dass alle Griffe im Kontakt mit dem Patienten behutsam und so flächig wie möglich gewählt werden. Techniken wie die Triggerpunktbehandlung müssen durch die deutlich dünnere Haut und die somit verletzlicheren darunter befindlichen Gefäße mit Bedacht und weit vorsichtiger eingesetzt werden als bei jüngeren Erwachsenen. Zeigt die Altershaut bereits eine verstärkte Neigung zu Hämatomen, sollten intensive Techniken komplett gemieden werden.

\section{Aktive Übungen}

Um einen weiteren Verlust an Fähigkeiten und Kraft zu vermeiden, ist der Erhalt der Muskulatur zum Hantieren und Greifen von Objekten eine wesentliche Zielsetzung. Dies kann während einer nötigen Immobilisation erfolgreich durch mentales Training erreicht werden [13].

Nach der Immobilisation gehören aktive Übungen zu den therapeutisch notwendigen Maßnahmen, ebenso wie eine gute Anleitung von Heimübungen für Patienten und Angehörige. Ein einfaches Beispiel hierfür ist die Eigentraktion am Handgelenk bei verminderter

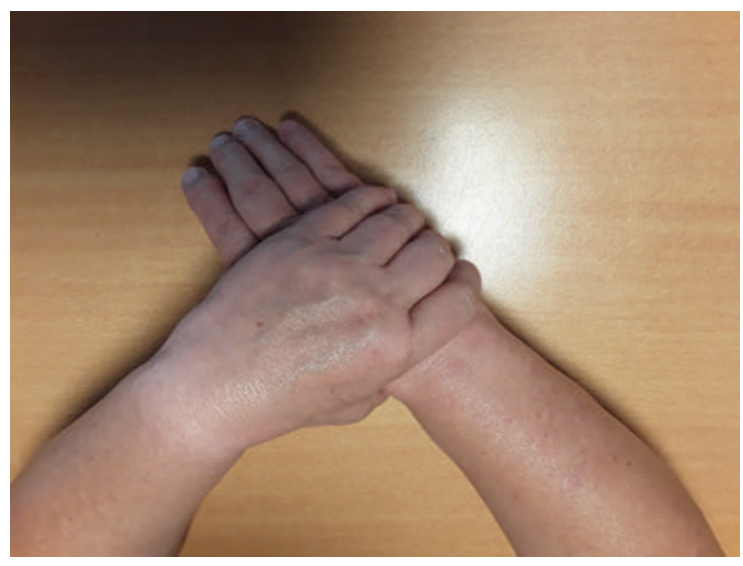

Abb. 3 Eigentraktion des Handgelenks. (Quelle: S. Wittek) 
Beweglichkeit des Gelenks nach Radiusfraktur ( A Abb. 3). Letztlich wird ein verbesserter Krafteinsatz auch der Knochendichte zugutekommen, da die vermehrte Krafteinwirkung auf den Knochen für diesen einen effektiven Bildungsreiz darstellt und den knöchernen Aufbau fördert. Ein weiterer wichtiger Aspekt ist die schnelle Wiederaufnahme von Alltagshandlungen und Bewegungen, die oftmals nur durch eine frühzeitige Hilfsmittelversorgung für die Dauer der Rekonvaleszenz zu erreichen ist.

\section{Beachtung des Sturzrisikos}

Durch Medikamenteneinnahme kann das Sturzrisiko begünstigt werden. Die Angaben zu relevanten Nebenwirkungen sind daher grob abzuschätzen. Insbesondere bei Umstellungen der Medikation können relevante Symptomlagen auftreten. Dazu gehören neu aufgetretener Schwindel, ein ständig trockener Mund, veränderte Wahrnehmung oder auch ein allgemeines Schwächegefühl. Bei der großen Menge an Medikamenten, die ältere Menschen einnehmen, kann es zu Verstärkungen einzelner Wirkungen kommen, die nicht beabsichtigt sind. Eine grobe Leitlinie ist, dass bei Patienten, die mehr als sechs Medikamente einnehmen müssen, der Plan ärztlicherseits überprüft werden sollte. Dies gilt insbesondere dann, wenn verstärkt unerwünschte Wirkungen auftreten, die die Alltagsfähigkeiten des Patienten einschränken.

\section{Den alternden Körper in Bewegung halten}

Bedenkt man die begünstigenden Faktoren vieler Abbauprozesse im Alter, so spielt dabei die Immobilisation eine große Rolle. Dabei ist es gleichgültig, ob es um ein vermindertes Bewegungsverhalten im Alltag oder um eine Folge einer Erkrankung geht. Für den Funktionserhalt aller am muskuloskelettalen System direkt beteiligten Strukturen sind der wichtigste Faktor die Bewegung und Belastung der Struktur.

Dies gilt sowohl für Arthrosen wie Heberden-, Bouchardoder Rhizarthrose als auch für eine Minderung der Muskelkraft. Für alte Menschen ist es zwar schwerer, sich an neue Bewegungsmuster zu gewöhnen, aber nicht unmöglich. Sie stellen eine Herausforderung, also einen Bildungsreiz, für das neuromuskuläre und kognitive System dar. Erleichternd für den Aufbau des Handgewölbes, der zur Entlastung des Daumensattelgelenks und zur Verbesserung der Griffkraft sinnvoll ist, kann mit kleinen Objekten gearbeitet werden, die beim Hantieren mit dem kleinen Finger fixiert werden sollen.

Die distale Radiusfraktur ist bei älteren Menschen oftmals ein Risikofaktor für den Beginn einer Osteoporose [12]. Dies ist dadurch bedingt, dass der Arm im Alter als Stützorgan benötigt wird. Fällt diese Funktion aufgrund einer Fraktur für mehrere Wochen aus, erfährt des Gesamtsystem weit weniger Belastung durch die eingeschränkte Gesamtmobilität des Patienten. Die Nachsorge von distalen Radiusfrakturen und die Behandlungsansätze sind

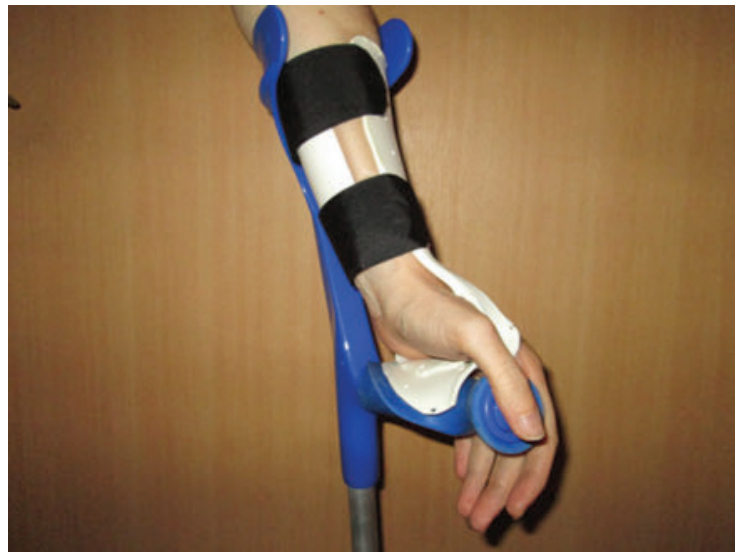

- Abb. 4 Dorsopalmare Handgelenksschiene mit Auflagesteg für den Griff der Stütze / des Stocks / des Rollators (Quelle: R. Groth)

zwar denen bei jüngeren Erwachsenen sehr ähnlich, doch gibt es auch ein paar wichtige Anpassungen: So gehört zur Nachsorge unbedingt nicht nur die Mobilisation der Gelenke, sondern vielmehr rückt der Funktionserhalt in den Vordergrund. Die Frage der Alltagsfunktionen bestimmt weit mehr das therapeutische Vorgehen als die vollständige Wiederherstellung. Es ergibt sich die Wahl, die Greifposition am Rollator, an Gehhilfen (Rollator mit Unterarmauflage) oder die Umleitung der Stützkräfte auf den proximalen Unterarm mittels Schiene zu verändern ( Abb.4). Hierzu wird allerdings eine ausreichende Stabilität der Haut benötigt, da diese trotz Polsterung einen Teil der Stützlast mittragen muss.

Oftmals gehört zu diesen Alltagsfunktionen eine schmerzfreie und stabile Stützfunktion, um den Gesamtorganismus aus einer Immobilisation zu nehmen und präventiv weiteren Frakturen vorbeugen zu können. Eine Schulung des Gleichgewichts und des Reaktionsvermögens gehört in das Gesamtpaket einer effektiven Sturzprävention. Dabei ist es wichtig, Bewegungsanforderungen in den Alltag des Patienten zu integrieren, sodass ein nachhaltiger Therapieerfolg möglich werden kann.

\section{Fazit}

Der Blick des Handtherapeuten muss ganzheitlich auf die Funktionsfähigkeit der verschiedenen Körpersysteme gerichtet sein und nicht allein auf die Behandlung der verletzten Struktur. An dieser wird mit denselben Techniken und in sehr ähnlichen zeitlichen Abläufen gearbeitet wie bei jüngeren Patienten. Angepasst werden sollte der Griff des Therapeuten, sodass dieser möglichst schonend mit Haut, Bindegewebe und Weichteilgewebe umgeht. Je punktueller der Griff, desto mehr Kraft benötigt der Therapeut. Die Krafteinwirkung auf die geschwächten Altersgewebe wird stärker, die dieser Last nicht mehr grundsätzlich standhalten können. 
Schnelle Bewegungen sind zu vermeiden, da sie vom neuromuskulären System nicht mehr in ausreichender Geschwindigkeit verarbeitet werden und Bindegewebsstrukturen sich nicht mehr ausreichend daran anpassen können. Belastungen sollten langsam und nach dem aktuellen Befinden des Patienten zu seinem Alltagsbedarf gesteigert werden, um den Strukturen die nötige Zeit zum Aufbau zu geben.

\section{Autorinnen / Autoren}

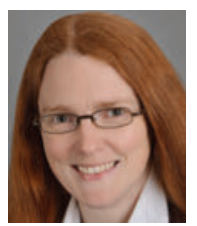

\section{Rebecca Groth}

Seit 2001 Ergotherapeutin (Bachelorabschluss 2006). Sie ist als zertifizierte Handtherapeutin im Rehazentrum Petzvalstraße in Braunschweig tätig und FH-Dozentin der Akademie für Handrehabilitation.

\section{Korrespondenzadresse}

Rebecca Groth

E-Mail: Reb.Groth@gmx.de

\section{Literatur}

[1] Arinci Incel N. Hand functions in geriatric conditions. In: Tuncay Duruöz M, ed. Hand Function: A Practical Guide to Assessment. New York: Springer; 2014

[2] van den Berg F. Grundlagen der Bindegewebsphysiologie. In: van den Berg F, Cabri JM, Voll M. Angewandte Physiologie Das Bindegewebe des Bewegungsapparates verstehen und beeinflussen. Stuttgart: Thieme, 2011

[3] Bundesministerium für Bildung und Forschung (BMBF). Medikamente im Alter. Im Internet: www.bmbf.de/pub/Medika mente_im_Alter.pdf; Stand: 08.2017
[4] Cooper C. Geriatric hand therapy. In: Cooper C, ed. Fundamentals of Hand Therapy. USA: Elsevier; 2007

[5] Gibson S. Progress in Pain Research and Management. Proceedings of the 10th World Congress on Pain 2002. Seattle: IASP Press; 2003

[6] Groth R. 63. Behandlung von Sturzfolgen beim geriatrischen Patienten. Ergotherapie-Kongress des DVE, 2018. Würzburg, 21.-23. Juni 2018

[7] Holt S, Schmiedl S, Thürmann PA. Priscus-Liste potenziell inadäquater Medikation für ältere Menschen. Im Internet: http: // priscus.net/download/PRISCUS-Liste_PRISCUS-TP3_2011.pdf ; Stand: 05.11.2019

[8] Kaye A, Baluch A, Scott J. Pain management in the elderly population: A review. The Ochsner Journal 2010; 10(3): 179-187

[9] Latienbacher S, Kunz M, Strate P et al. Age effects on pain thresholds, temporal summation and spatial summation of heat and pressure pain. Pain 2005; 115(3): 410-418

[10] Neyedli H, Dampaio-Baptista C, Kirman M et al. Increasing lateralized motor activity in younger and older adults using real-time fMRI during executed movements. Neuroscience 2018; 378: 165-174

[11] Tautenhahn J, Piatek S. Polyurethanschaumstoffe / Hydropolymere. In: Lippert H, Hrsg. Wundatlas. 3. vollständig überarbeitete und erweiterte Auflage. Stuttgart: Thieme; 2012. doi:10.1055/b-002-35728

[12] Oyen J, Brudvik C, Gjesdal CG et al. Osteoporosis as a risk factor for distal radius fractures. J Bone Joint Surg Am 2011; 93 (4): 348-356

[13] Scott N, Korbus H. Preventing functional loss during immobilization after osteoporotic wrist fractures in elderly patients: A randomized clinical trial. BMC Muskuloskelet Disord 2014; 15: 287. doi:10.1186/1471-2474-15-287

[14] Stevens JC, Foulke E, Patterson MQ. Tactile acuity, aging, and braille reading in long-term blindness. J Experimental Psych 1996; 2(2): 91-106 